\title{
TRIZ AND COMPUTER AIDED INVENTING
}

\author{
Sergei Ikovenko \\ Professor Adjunctis (MIT, Cambridge, USA), International TRIZ Association (MA TRIZ)
}

Abstract: $\quad$ TRIZ (Theory for Inventive Problem Solving) being one of the most powerful inventing methodologies is sophisticated and requires considerable time and efforts to master. There have been and are multiple projects on developing various software packages that would make TRIZ usage and applications more user-friendly and shorten the TRIZ learning curve. Software packages that address this issue are one of the first computer aided inventing tools known. This direction of CAI has become much more active when TRIZ started its integration with other engineering methods - Value Engineering Analysis (VEA), Root-Cause Analysis (RCA), Lean Thinking, Six Sigma, Functional Analysis and others. Several software packages assist engineers with documenting data, building component and function models, and automatically calculating function rank of the components of the system. Performing a function-based information search is one of the tasks where computerized support is helping if finding new functions for improving complex systems. The next step is to identify other systems in which similar functions are performed better. The principles used to achieve similar functions in other fields can then be adapted to improve the system at hand. Developing powerful tools for Function Based Information Search to enable the substitution of inventing problems with adaptation problems is predicted to be one of major directions of CAI development

Key words: Computer-Aided Inventing, TRIZ, TRIZ++, Root-Cause Analysis, Evolutionary Analysis. 


\section{INTRODUCTION}

TRIZ (Theory for Inventive Problem Solving) being one of the most powerful inventing methodologies is sophisticated and requires considerable time and efforts to master.

There have been and are multiple projects on developing various software packages that would make TRIZ usage and applications more userfriendly and shorten the TRIZ learning curve.

This direction of CAI has become much more active when TRIZ started its integration with other engineering methods - Value Engineering Analysis (VEA), Root-Cause Analysis (RCA), Lean Thinking, Six Sigma and others.

TRIZ is a scientifically-based and empirically-derived method that originated for the analysis of the world patent collection. Its strongest side is in the Conceptual Stage of design, while the Analytical Stage is not completely covered by TRIZ.

VEA and RCN, for example, on the contrary are extremely effective for the analysis, but lack the heuristic power of TRIZ. That is why the combination of analytical and concept generating methods is leading to increasing effectiveness and higher quality of engineering solutions.

An inevitable stumbling block, a contradiction on this path is continually growing complexity and sophistication of the combined methods. CAI looks like that magic bridge that will allow getting the benefits of both worlds the effectiveness of the combined methods and relatively short learning curve and ease of their usage.

Merging TRIZ with other methods gave birth to several integrated methodologies based on TRIZ: ITD, I-TRIZ, TRIZ++, etc. It opens new horizons for CAI development to cover all the parts of those methods, both analytical and concept generating. As an example, this direction of CAI is illustrated on "TRIZ++" methodology.

\section{TRIZ ++ PROBLEM SOLVING APPROACH}

The TRIZ++ methodology offers a powerful and systematic approach for solving technical problems and improving engineering systems and manufacturing processes. Furthermore, when a problem is solved or a system is improved, the value of the engineering system or process is always increased when the TRIZ++ methodology is used. Value is defined as benefits (for example, improved functional performance, increased productivity, accuracy, consumer acceptance, etc.) divided by generalized 
costs.1 One of the most important goals of TRIZ++ is to develop breakthrough solutions that significantly improve the value of a system, as opposed to offering compromise solutions.

The TRIZ++ approach is based upon clearly defining and understanding the main and supporting functions of a system and the underlying problems that must be solved to improve the system. If the functions of a system are clearly understood, harmful and non-useful functions can be eliminated and engineering contradictions within the system can be identified and resolved more easily. The goals of a project can be achieved with much less effort. One of the biggest traps that problem solvers often fall into is that they attempt to solve the wrong problem, often trying to eliminate the symptoms instead of the underlying root problems. Applying a functional approach to problem solving helps to avoid this pitfall.

In addition to its function-oriented approach, the TRIZ++ methodology is based on two other important principles. The first is that many engineering problems have already been solved elsewhere, but in fields that are remote from the one at hand. A function-based understanding of a system makes it much easier to search other fields for possible solutions. This approach also allows problem solvers to overcome psychological inertia and to formulate creative and unexpected solutions.

The second important principle is that future directions of development of an engineering system or a process can be predicted from predetermined trends. The Russian scientist Genrich Altshuller ${ }^{1}$ and his followers identified these Trends of Engineering System Evolution (TESE) after analysis of more than three million patents. With knowledge of these Trends, the problem solver knows where to search and how to develop solutions that correspond to the natural evolutionary development of the system to be improved.

The TRIZ++ methodology can be divided into two basic phases: the Analysis and Problem Statement Phase and the Conceptual Phase. The purpose of the Analysis and Problem Statement Phase is to identify key/root problems that must be solved to improve the system and achieve the goals of the project. The purpose of the Conceptual Phase is to develop and substantiate concepts that solve the key/root problems identified in the Analysis Phase. These phases are described in greater detail below.

1 Generalized costs include not only the dollar value assigned to the elements that execute a given function, but also the qualitative "costs" of implementing the function, for example, problems associated with implementation. The following three paths can be used alone or in combination to increase value: (1) improved efficiency in functional performance; (2) introduction of new functions; and, (3) cost reduction. 


\section{ANALYTICAL AND PROBLEM STATEMENT PHASE}

During the Analysis and Problem Statement Phase (see below), a set of TRIZ++ tools is systematically applied to analyze the system functions. This allows the problem solver to identify the key/root problems that must be solved to achieve the goals of the project and to increase the value of the system. These tools include Benchmarking, Function Analysis, Trimming Technique, Substance and Energy Flows Analysis, Cause-Effect Chains Analysis, a function-based information search, and S-Curve Analysis.

The power of these tools lies in their ability to help identify the key/root problems of the system, as opposed to perceived problems or superficial symptoms. Many problem solvers make the common mistake of trying to solve the wrong problem or trying to eliminate symptoms without addressing fundamental underlying problems. Upon completion of this phase, a set of clearly defined problem statements is articulated (Fig 1). These are the key problems that must be solved to achieve the goals of the project. These problem statements are specifically formulated so the TRIZ++ problem solution tools can be effectively applied during the Conceptual Phase.

\subsection{Function Analysis}

The purpose of Function Analysis is to build a function model of the system. The first step is to determine and to represent the main and the supporting functions of the system. Next, functions are identified as harmful or useful. Useful functions are further defined as normal, excessive, or insufficient. The important parameters of the useful functions are also defined. Finally, functions are ranked according to their importance.

Several software packages assist engineers with documenting data, building component and function models, and automatically calculating function rank of the components of the system.

\subsection{Substance and Energy Flows Analysis}

Substance and Energy Flows Analysis is used to identify areas in the system where substances and/or energy are lost, not used efficiently, or have harmful effects. The result of this analysis is a list of disadvantages in the system associated with substance and energy flows.

There have been some attempts, but for the time being, that part of the analysis is a challenge for CAI. 


\subsection{Cause-Effect Chains Analysis}

Our intuition usually tells us that the larger the number of problems associated with an engineering system or a process, the harder it will be to eliminate them all and to improve the system. However, this is often not the case. One problem can create another problem, which creates another problem and so on. If the root problems are identified and eliminated, then all the associated problems, or a significant number of them, will disappear. Furthermore, breakthrough solutions often occur when root problems are solved. Cause-Effect Chains Analysis is one of the tools used to identify root problems.

Cause-effect chains are built from the disadvantages identified during Function Analysis, Trimming, and Substance and Energy Flow Analysis. These chains may branch and/or intersect. They end with known disadvantages associated with high costs, low productive capacity, poor quality, and other shortcomings. If a key disadvantage is eliminated, all subsequent disadvantages in its specific chain will be eliminated. Key disadvantages are usually found at the beginning of a cause-effect chain or at the intersection of two or more chains.

RCA-based software packages and modules have been developed and are being used both independently and a part of VEA/RCA/TRIZ software suites. 


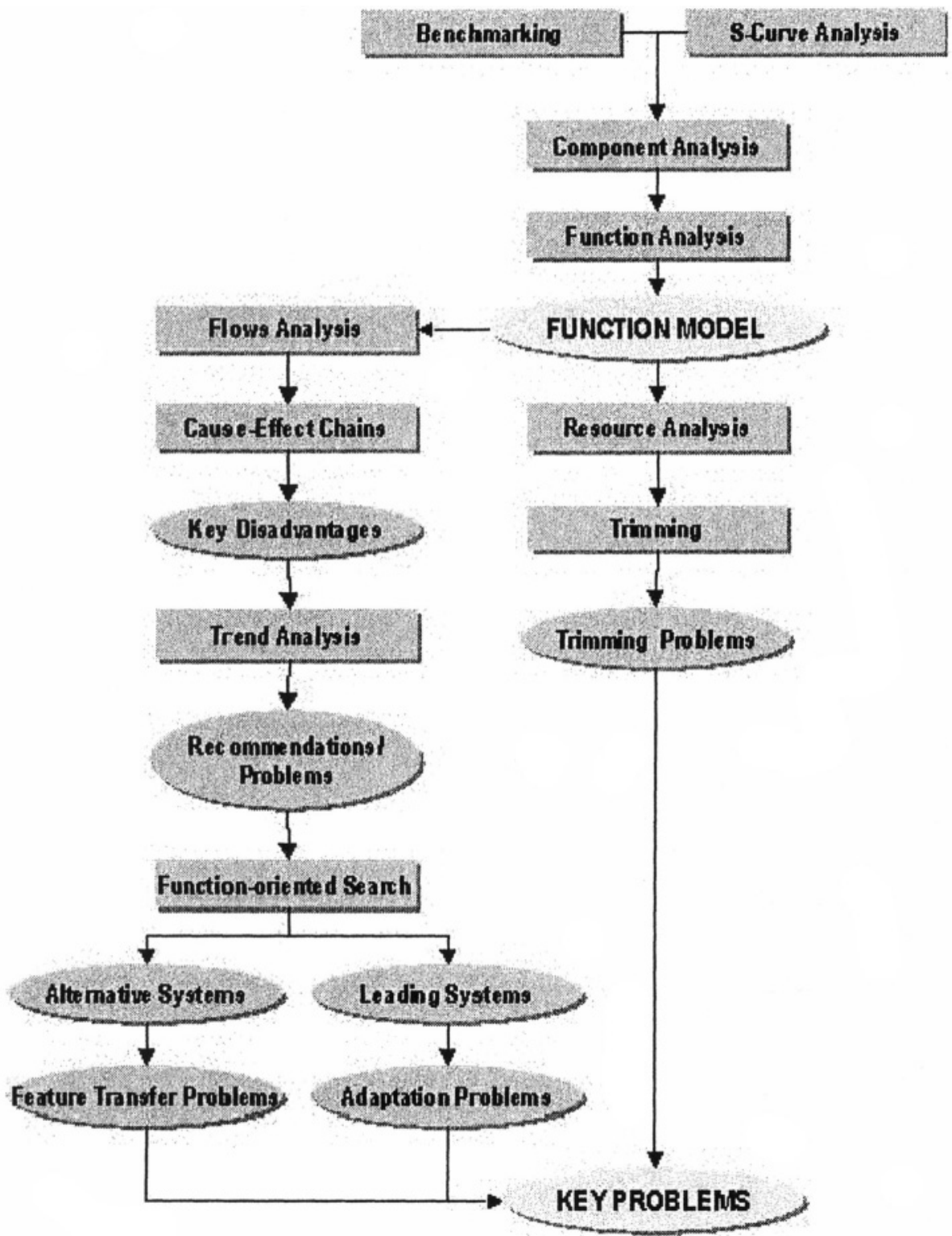

Figure 1. Flow Diagram for Key Problem Solving

\section{$3.4 \quad$ Trimming}

The purpose of Trimming is to improve the system by eliminating harmful and low-ranked functions. The result of Trimming is a set of newly 
formulated problem statements. These statements identify the problems that must be solved to eliminate the harmful and low-ranked functions and their associated components. The key is to preserve the important useful functions associated with the components that are eliminated.

Several CAI products cover this part of the method.

\subsection{Function-Based Information Search}

The purpose of a function-based information search is to identify solutions to problems in areas of science and engineering that are farremoved from the system at hand. Borrowing ideas or practical solutions from other fields of knowledge can be very effective. Often scientists or engineers who are experts in the field where a problem lies have little or no knowledge of the field(s) where solutions lie. Consequently, finding successful solutions is usually a haphazard process.

The key to performing a function-based information search is first to identify specific functions in the system that should be improved or modified. The next step is to identify other systems in which similar functions are performed better. The principles used to achieve similar functions in other fields can then be adapted to improve the system at hand.

Software packages that address this issue are effective web search and information procession tools. The most advance direction of Function-Based Information Search is Semantic Technologies.

\subsection{Feature Transfer}

In addition to TRIZ, Feature Transfer is used to help develop concepts. This tool is designed to formulate ways of combining the advantages and best features of competing engineering systems (products and processes) into a single system. The power of Feature Transfer is not that the specific elements of one engineering system are "mechanistically" transferred to another system, but rather, that the underlying reasons why a specific feature works well are analyzed and applied to the system under development.

Feature Transfer ${ }^{\mathrm{TM}}$ approach is a further development of a technique known among design methods as Pugh Concept. A number of software packages allow using this approach.

\subsection{Evolutionary Analysis}

Evolutionary Analysis is used to determine the general direction in which a system will naturally evolve to become an ideal system. This information is used to help determine the best directions and concepts for improving the 
system. In particular, Evolutionary Analysis will help to determine if a system can be substantially improved based on the current principle(s) of operation or if fundamentally, new principle(s) of operation are required to achieve substantial improvements.

Evolutionary Analysis is based upon the Trends of Engineering System Evolution. According to the Trends of Engineering System Evolution, an engineering system passes through four consecutive stages as it evolves: (1) slow development; (2) rapid growth; (3) stabilization; and (4) decline. This evolution over time can be represented as an S-shaped curve. Once a system has reached the fourth stage of decline, fundamentally new principles of operation are required to substantially improve the system.

CAI development in this direction is rudimentary and has had a merely visualization objective.

\subsection{Results of the Analysis and Problem Statement Phase}

The primary result of the Analysis and Problem Statement Phase is a set of key problems that must be solved in order to achieve the goals of the project. Very often, the results of the Analysis and Problem Statement Phase also include a redefinition of the goals of the project and fundamental changes to the initially proposed methods of achieving these goals. This is because the initial problem statements are significantly better understood as a result of the analysis.

\section{CONCEPTUAL PHASE}

The purpose of the Conceptual and Substantiation Phases (see diagram below) is to develop feasible concepts that will solve the key problems identified in the Analysis and Problem Statement Phase. A conceptual idea is an idea that is supported by references to scientific or technological sources, expert opinions, and/or known experimental results. 


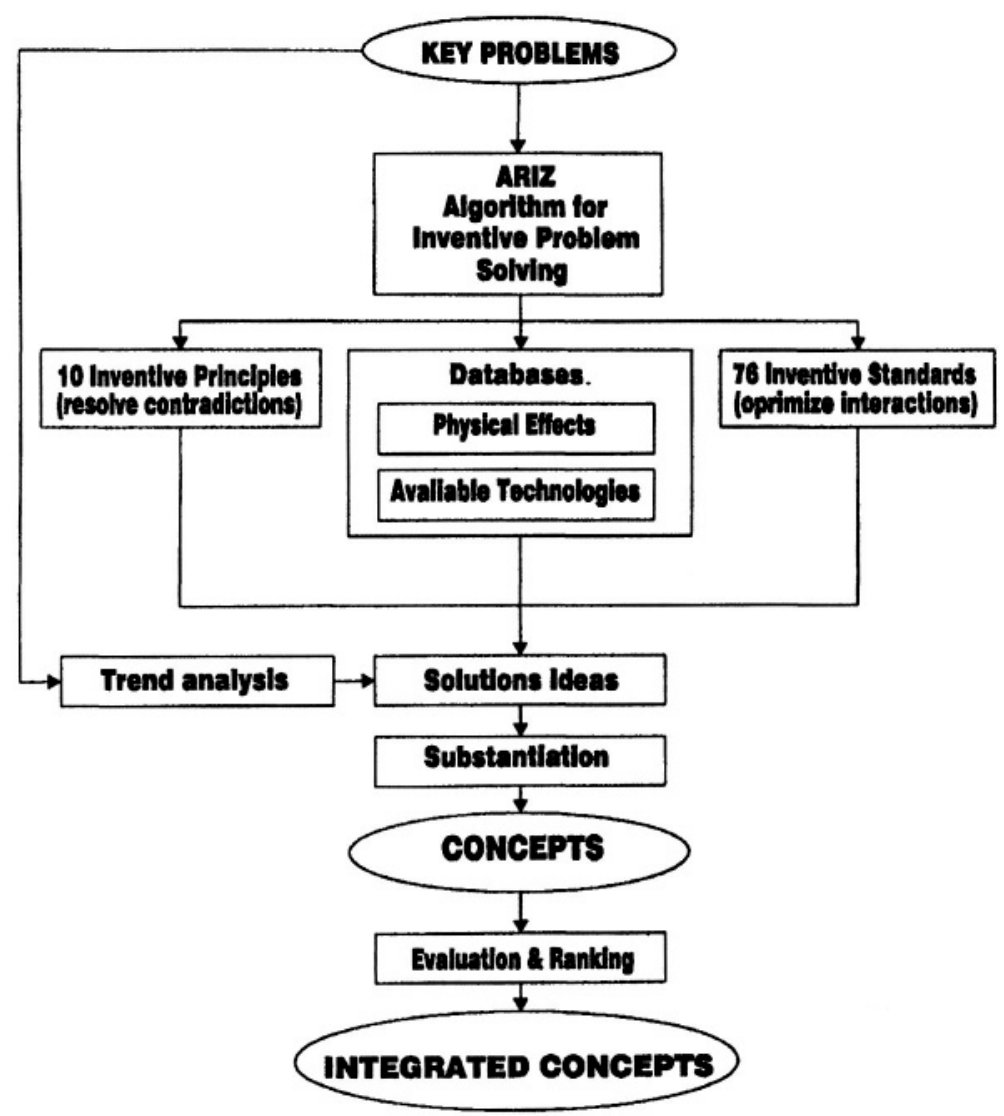

Figure 2. Flow Diagram for Key Problem Solving

Conceptual Phase includes all the types of problem modeling in TRIZ: engineering contradictions, Substance-Field Modeling, Physical Contradictions, and all the TRIZ 
Tools to work with these models: Contradiction Matrix, the System of Standard Inventive Solutions and a database of scientific and engineering effects.

This part of the design process has been extensively covered by multiple software packages, although there are challenges still.

\subsection{Concept Ranking and Compatibility}

Some of the final concepts will be better at achieving certain results than others. However, cost, time, difficulty of implementation, and resource availability must be taken into account when assigning value to the concepts. Concepts are ranked based on a set of project-dependent criteria.

Some concepts may be implemented in combination and others may not be. In certain cases, the combined implementation of two or more concepts may offer extremely powerful solutions. A matrix of possible combinations in which the individual concepts could be implemented is also developed.

Multiple software packages address this part of the design.

\section{CONCLUSIONS}

The above-described TRIZ++ Methodology shows that there are a lot of gaps that CAI has not covered. I-TRIZ Methodology has some of its parts supported by the software while some parts are simply manual.

A new approach in TRIZ application and CAI has become obvious. Inventive solutions though being powerful always require proof of concept, substantiation, verification, tests. It makes the Implementation Stage long or the inventive solution can be abandoned altogether. TRIZ++ address this issue in a pretty elegant way. Using very effective Function Based Information Search, they find an existing technology that would solve problems identified in the Analysis Phase. As a rule, the technology comes from a totally different area and requires adaptation. However, the proof of concept is unnecessary and the verification stage is much shorter for solving an INVENTIVE problem is substituted with solving a VERYFICATION problem. To make this happen powerful tools for Function Based Information Tools are crucial.

Therefore, it is possible to predict two major directions of CAI development:

Computerizing "gaps" of classical TRIZ and integrated suites of TRIZ and other methods 
Developing powerful tools for Function Based Information Search, to enable the substitution of inventing problems with adaptation problems. A most probable technology for such a tool is semantic technology

\section{REFERENCES}

1. Altshuller, G.S. Creativity as an exact science: the theory of the solution of inventive problems / New York: Gordon and Breach, 1995

2. S. Litvin. TRIZ++ - an Advanced Approach to TRIZ. ENITechnologie Conference Proceerings, Milan, 2003. S. Ikovenko. TRIZ and Semantic Technologies in Innovation and Knowledge Management. CROInfo2004, Zagreb, 2004. 